\section{Uso exclusivo de álcool e em associação a outras drogas entre motoristas de caminhão que trafegam por rodovias do Estado de São Paulo, Brasil: um estudo transversal}

\author{
Alcohol use alone and in combination with other \\ drugs among truck drivers on highways in São \\ Paulo State, Brazil: a cross-sectional study \\ Consumo exclusivo del alcohol, en combinación \\ con otras drogas, entre los conductores que \\ transitan por las carreteras del Estado de São \\ Paulo, Brasil: un estudio transversal
}

\author{
${ }^{1}$ Faculdade de Medicina, \\ Universidade de São Paulo, \\ São Paulo, Brasil. \\ Correspondência \\ L. G. Oliveira \\ Departamento de Medicina \\ Legal, Ética Médica e \\ Medicina Social e do \\ Trabalho, Faculdade de \\ Medicina, Universidade \\ de São Paulo. \\ Av. Dr. Arnaldo 455, São \\ Paulo, SP 01246-903, Brasil. \\ lucgoliver@gmail.com
}

\begin{abstract}
Traffic accidents cause more than a million deaths per year worldwide. Brazil is one of the leading countries in traffic accidents. Since alcohol is a known etiological factor for traffic accidents, the pattern of alcohol use alone and in combination with other drugs was assessed in a convenience sample of 684 truck drivers in São Paulo State, Brazil. 67.3\% of participants reported alcohol use in the previous 30 days, $34.6 \%$ reported heavy drinking, $26 \%$ binge drinking, and 9.2\% were at risk of developing alcohol addiction. Furthermore, 54.6\% reported multiple drug use, mostly alcohol with tobacco and energy drinks. Truck drivers that reported multiple drug use showed heavier alcohol use than those who consumed only alcohol. Drinking and driving is problematic and can be aggravated by multiple drug use. The authors thus suggest monitoring the issue, which creates serious problems for users and society as a whole.
\end{abstract}

Traffic Accidents; Alcoholism; Alcoholic Beverages; Cross-sectional Studies
Kae Leopoldo 1

Vilma Leyton 1

Lucio Garcia de Oliveira ${ }^{1}$

\section{Resumo}

Os acidentes de trânsito têm causado mais de um milhão de mortes no mundo. O Brasil é um dos países recordistas quanto a esse indicador. Como o consumo de álcool é fator etiológico para o acontecimento de acidentes de trânsito, buscou-se estimar o padrão de uso de álcool e o uso múltiplo de álcool e outras drogas em uma amostra de conveniência de 684 motoristas de caminhão no Estado de São Paulo, Brasil. Observou-se que, nos trinta dias prévios à entrevista, 67,3\% dos participantes usaram álcool, 34,6\% de forma pesada, 26\% como binge drinking e 9,2\% estavam sob o risco de desenvolver dependência. Ainda, 54,6\% deles relataram o uso múltiplo de álcool e outras drogas, tendo usado álcool predominantemente com tabaco e energéticos. Os participantes que relataram uso múltiplo de álcool e outras drogas apresentaram consumo mais pesado de álcool em relação àqueles que ingeriram apenas álcool. Em linhas gerais, o uso de drogas é um problema no contexto de trânsito e pode piorar sob a condição de uso múltiplo de álcool e outras drogas. Assim, os autores sugerem a vigilância do tema, dado que esse quadro gera, potencialmente, não só sérios problemas à saúde do usuário, mas também à sociedade como um todo.

Acidentes de Trânsito; Alcoolismo; Bebidas Alcoólicas; Estudos Transversais 


\section{Introdução}

A Organização Mundial da Saúde (OMS) apontou que os acidentes de trânsito causaram, em 2010, cerca de 1,24 milhões de mortes e feriu de 20 milhões a 50 milhões de pessoas no mundo, gerando um ônus de $1 \%$ a $2 \%$ do produto interno bruto (PIB) dos países de renda baixa e média 1 .

No Brasil, no ano de 2012, houve 46.051 mortes (Departamento de Informática do SUS. Estatísticas vitais. http://www2.datasus.gov.br/ DATASUS/index.php?area=0205, acessado em 17/Nov/2014) e 619 mil pessoas foram feridas em decorrência de acidentes de trânsito 2 , onerando os cofres públicos em 22 bilhões de Reais, o correspondente a $1,2 \%$ do PIB brasileiro 3. Nesse cenário, as rodovias do Estado de São Paulo concentram o maior número de acidentes de trânsito, totalizando mais de 70 mil acidentes e 20 mil vítimas por ano ${ }^{3}$. Quanto ao tipo de veículo, os caminhões respondem por, aproximadamente, $30 \%$ dessas ocorrências nas rodovias brasileiras (Departamento Nacional de Infraestrutura de Transportes. Estatísticas de acidentes. http://www.dnit.gov.br/rodovias/operacoesrodoviarias/estatisticas-de-acidentes, acessado em 17/Nov/2014).

Dirigir sob o efeito de álcool é uma das principais causas de acidentes de trânsito 1 , de tal forma que a ingestão de poucas doses alcoólicas já é suficiente para aumentar a predisposição do condutor para emitir algum comportamento de risco 4 . No Brasil, é preocupante notar que quase $40 \%$ da população relataram ter dirigido ao menos uma vez depois de ter bebido 5 .

Especificamente entre os motoristas de caminhão, estudos sobre o uso de álcool e outras substâncias psicoativas ainda são incipientes 6 . Uma revisão sistemática da literatura apontou recentemente que $54,3 \%$ dos motoristas de caminhão brasileiros relataram usar álcool, enquanto $3,6 \%$ deles tinham algum nível de álcool no sangue, conforme os resultados da análise toxicológica de amostras biológicas 6, sugerindo, então, que esses últimos estivessem dirigindo sob o efeito de álcool. Entretanto, os poucos estudos que avaliaram o uso de álcool entre os motoristas de caminhão deixaram de caracterizar o padrão de uso, assim como não identificaram quantos destes estariam sob o risco de desenvolver abuso ou dependência. Essa informação é imprescindível, já que reincidentes do comportamento de beber e dirigir fazem uso pesado de álcool 7, necessitando de intervenções focadas no sujeito.

Some-se a esse quadro o fato de que o uso de álcool pode dificultar ou impossibilitar a atividade laboral dos motoristas de caminhão, uma vez que os transtornos mentais e comportamentais relacionados ao consumo de álcool são os que causam maior número de afastamentos do trabalho e aposentadorias precoces 8 . Nesse contexto, prioridade deve ser dada à investigação da tendência atual do uso múltiplo de drogas 9,10,11, em que usuários de álcool usam-no juntamente a outras substâncias psicoativas, ao mesmo tempo ou não, com finalidades diversas 10 . Preocupa notar que o uso múltiplo de drogas agrava a emissão de comportamentos de risco no trânsito ${ }^{12}$, tornando o condutor mais predisposto ao acontecimento de acidentes de trânsito. Embora a existência do uso múltiplo de drogas já tenha sido citada entre motoristas de caminhão 13 , detalhamentos são necessários.

Em março de 2010, a Assembleia Geral das Nações Unidas proclamou o período de 2011 a 2020 como a Década de Ação pela Segurança no Trânsito, que tem por objetivo reduzir $50 \%$ da mortalidade mundial no trânsito 14 . O Brasil é um dos países signatários e também se propôs a essa meta mundial. Dessa forma, conhecer o padrão de uso de álcool e sua associação a outras drogas entre motoristas de caminhão é subsídio imprescindível para que as autoridades públicas e competentes elaborem medidas de intervenção e políticas públicas que venham a reduzir os acidentes de trânsito pela categoria. Ademais, em contexto nacional, a realização de estudos sobre o uso de álcool entre os motoristas é essencial à compreensão e, sobretudo, ao cumprimento das leis que coíbem o comportamento de beber e dirigir, como a Lei no 11.705/2008 15 (conhecida por Lei Seca) e a Lei no 12.706/2012 16, que estabelecem punições severas aos infratores.

Portanto, de forma inédita, o presente trabalho avalia o padrão de uso de álcool e a existência de uso múltiplo de drogas entre motoristas de caminhão, com a identificação das substâncias psicoativas mais frequentemente envolvidas nessa situação, assunto que não foi tratado diretamente por estudos prévios, tampouco por levantamentos estatísticos. Finalmente, levanta-se a hipótese de que usuários de múltiplas drogas consumiriam mais álcool do que usuários exclusivos de álcool, questão que também é foco deste estudo.

\section{Métodos}

Este artigo é parte integrante de um estudo epidemiológico, exploratório, observacional, do tipo transversal, aprovado pelo Comitê de Ética em Pesquisa (CEP) da Faculdade de Medicina, Universidade de São Paulo (FMUSP; protocolo no $377 / 11$ ). A pesquisa foi realizada no período de junho de 2012 a setembro de 2013, sendo 
contemplada com recursos financeiros da Fundação de Amparo à Pesquisa do Estado de São Paulo (FAPESP), Programa Auxílio Pesquisa Jovem Pesquisador (processo no 2011/11682-0).

\section{Participantes}

Uma amostra não probabilística de 684 motoristas de caminhão que circulavam por três rodovias do Estado de São Paulo (Presidente Dutra, Fernão Dias e Cônego Domênico Rangoni) foi recrutada em postos de atendimento das entidades civis Serviço Social do Transporte (SEST), Serviço Nacional de Aprendizagem do Transporte (SENAT. http://www.sestsenat.org.br/Pagi nas/Index.aspx). Como a pesquisa que originou o presente estudo pretendeu avaliar os efeitos do uso de substâncias psicoativas sobre o funcionamento de atenção de motoristas de caminhão, os sujeitos que apresentassem pelo menos uma condição de saúde que interferisse nessa avaliação foram excluídos da amostra, a citar: (a) ter dificuldade para a visualização de cores; (b) estar sob o uso terapêutico de medicamentos psicoativos; (c) relatar o acontecimento de traumatismo cranioencefálico (TCE) na vida; (d) ter sofrido algum episódio com perda de consciência na vida; (e) ter histórico de doenças neurológicas, assim como (f) de soropositividade para HIV.

\section{Instrumentos}

\section{- Informações sociodemográficas, antropométricas e ocupacionais}

Um instrumento de pesquisa estruturado, já validado por Leyton et al. 17 para uso com motoristas de caminhão no Brasil, foi aplicado a fim de registrar as informações demográficas (idade, estado civil, nível de escolaridade, entre outras) e ocupacionais (tipo de vínculo empregatício, carga diária de trabalho, distância percorrida, entre outras) dos participantes. Também foram registradas suas informações antropométricas: peso, altura, índice de massa corporal (IMC), circunferência cervical e abdominal, pressão arterial (PA), frequência cardíaca (bpm), entre outras. Como parte do instrumento, o participante foi questionado sobre a prática de atividade física e existência de agravos de saúde, tais como (a) hipertensão arterial (HAS); (b) diabetes mellitus; (c) dislipidemia e (d) histórico familiar de doença arterial coronariana (DAC); perguntou-se ainda (e) se fazia uso de medicamentos não psicoativos e (f) qual teria sido a data da última consulta médica. Como as informações antropométricas não fazem parte do escopo deste estudo, não são aqui descritas em detalhes.

\section{- Informações sobre uso de álcool e outras drogas}

Ainda como parte do instrumento de pesquisa, os participantes foram solicitados a responder sobre a experiência pessoal com o uso de álcool, tabaco e outras drogas, conforme o uso na vida (definido como o uso experimental, ou seja, "pelo menos uma vez na vida"), nos últimos 12 meses (no ano, ou seja, "pelo menos uma vez nos 12 meses que antecederam a entrevista") e nos últimos 30 dias 18 . Especificamente sobre o uso de álcool, se foi positivo nos últimos 30 dias, determinou-se, então, a frequência e, sobretudo, a quantidade de uso em uma sessão típica (em termos do número de doses-padrão de álcool) e na ocasião de maior consumo. Posteriormente, as variáveis de frequência e quantidade de uso de álcool foram combinadas para determinar o padrão de consumo da substância nos últimos 30 dias. Então, o padrão de uso de álcool foi categorizado em leve, moderado e pesado, de acordo com os critérios adotados pela pesquisa Gender, Alcohol and Culture: An International Study (GENACIS) 19, da OMS. Ainda nesse sentido, os participantes foram questionados sobre o uso de álcool no padrão beber pesado episódico (BPE ou binge drinking), definido, para homens, como o uso de cinco ou mais doses-padrão de álcool por ocasião, dentro de um período de duas horas 20. Os critérios do Teste de Triagem do Envolvimento com Álcool, Cigarro e Outras Substâncias (ASSISTWHO, versão 3.1) 21 também foram incluídos no instrumento de pesquisa, a fim de avaliar o nível de risco para o desenvolvimento de dependência, em associação ao uso de álcool, categorizado em: (a) "risco baixo" (uso ocasional ou não problemático; até 10 pontos); (b) "risco moderado" (uso regular que necessita de intervenção breve; de 11 a 26 pontos) e (c) "risco alto" (uso frequente e problemático, indicativo de que o indivíduo já deve ser encaminhado a um programa especializado de tratamento; 27 ou mais pontos). Como as frequências de uso de "risco alto" foram baixas, as pontuações referentes a "risco moderado" e "risco alto" foram agrupadas (acima de 11 pontos), visando a descrever, no geral, o uso nocivo de álcool. Finalmente, as medidas de uso na vida, no ano e no mês foram empregadas para a avaliação da frequência do uso múltiplo de drogas entre os participantes, mediante procedimentos já descritos por Oliveira et al. 11. Nesse sentido, duas categorias de uso múltiplo foram investigadas: (a) uso múltiplo de drogas do tipo concorrente (CPU), que consiste no uso de duas ou mais substâncias psicoativas em ocasiões diferentes (dentro de um período de um mês, um ano ou qualquer outro intervalo de tempo), e 
(b) uso múltiplo de drogas do tipo simultâneo (SPU), que envolve o emprego de duas ou mais substâncias em uma mesma sessão de uso 9,10. Ambas as categorias de uso múltiplo de drogas tiveram como foco o uso de álcool (combinação de álcool a outras drogas), já que é a substância mais comumente envolvida nessa situação 22 .

\section{Procedimentos}

Todos os motoristas de caminhão que estavam à disposição nos postos de atendimento da SESTSENAT foram abordados por um recrutador da equipe, que, então, informava os potenciais participantes sobre a realização da pesquisa naquele dia, horário e posto de atendimento. Caso consentissem em participar, os motoristas eram então encaminhados a entrevistadores que explicaram os objetivos do estudo, o tempo necessário para responder aos instrumentos de pesquisa, ressaltando os princípios de voluntariedade e confidencialidade da informação. O Termo de Consentimento Livre e Esclarecido foi lido pelos entrevistadores e, quando de acordo, assinado pelos motoristas. Em contrapartida, se o motorista declinava de participar do estudo, suas informações de gênero, idade e motivo de recusa foram anotadas. Entre aqueles que aceitaram participar, o entrevistador aplicou o instrumento de pesquisa na íntegra, com a solicitação das informações supracitadas. Finalizado o preenchimento do questionário, o participante foi encaminhado à avaliação antropométrica por profissionais de saúde do Departamento de Medicina Legal, Ética Médica e Medicina Social e do Trabalho, da FMUSP. Todos os participantes foram avaliados individualmente, em ambientes fechados, seguros e silenciosos, cujo uso havia sido destinado exclusivamente à realização das atividades desta pesquisa.

\section{Análise dos dados}

Todos os questionários foram submetidos a procedimentos de digitação dupla. O banco de dados foi construído no software Epi Info versão 6.0 (Centers for Disease Control and Prevention, Atlanta, Estados Unidos). Checagens de consistência das informações e correções pertinentes foram realizadas. Posteriormente, os dados foram transferidos e analisados no programa Stata versão 11.2 (StataCorp LP, College Station, Estados Unidos). Procedeu-se, em seguida, à análise descritiva das variáveis. As variáveis categóricas foram expressas em porcentagens (\%; intervalo de $95 \%$ de confiança - IC95\%) e as variáveis numéricas, por meio de média \pm desvio padrão. Por vezes, algumas das variáveis numéricas foram categorizadas conforme a mediana. Para a avaliação das possíveis diferenças de padrão de consumo de álcool entre usuários apenas de álcool e usuários de múltiplas drogas, as variáveis categóricas foram comparadas valendo-se do teste de $\chi^{2}$ de Pearson e as variáveis numéricas, mediante o teste de Kruskal Wallis ou Mann-Whitney. A hipótese nula foi rejeitada ao nível de $\mathrm{p}<0,05$.

\section{Resultados}

\section{Dados sociodemográficos}

Aceitaram participar do estudo 684 motoristas de caminhão, 149 (22\%) dos quais foram excluídos por terem preenchido pelo menos um dos critérios de exclusão. Dos participantes excluídos, a maioria $(76,9 \%)$ preencheu apenas um critério de exclusão e os demais $(23,1 \%)$ dois critérios ou mais. Dentre estes, ter tido episódio de perda de consciência foi o mais prevalente (46,3\%), seguido do acontecimento de episódio de TCE $(27,9 \%)$. Assim, fazem parte desta pesquisa as informações referentes aos 535 motoristas restantes.

Os participantes têm idade média de 37,8 \pm 7,78 anos, 8,6 $\pm 2,28$ anos de escolaridade e, em sua maioria $(74,7 \%)$, declararam-se casados ou em situação marital. Quanto ao vínculo empregatício, 60,9\% dos participantes estavam contratados no momento da entrevista e tinham uma média de 12,5 $\pm 8,1$ anos de experiência como motoristas profissionais. A maioria $(60,2 \%)$ trabalhava em dois turnos (diurno e noturno), dirigia uma média de 12,1 \pm 3,88 horas diárias, percorrendo uma distância média de 1.127,3 \pm $1.020,84 \mathrm{~km}$ (Tabela 1).

\section{Sobre o uso de álcool}

Dos participantes, 95,7\% relataram ter consumido álcool ao menos uma vez na vida; $77,6 \%$, no ano; $67,3 \%$, nos trinta dias prévios à entrevista. Dentre aqueles que relataram ter bebido nos últimos trinta dias, $61,3 \%$ referiram fazê-lo com uma frequência pelo menos semanal. Em termos da quantidade ingerida de álcool, os participantes relataram o consumo médio de 6,4 doses alcoólicas por ocasião de uso e, quando solicitados a responder sobre o consumo máximo por ocasião, disseram ter ingerido uma média de 8,2 doses alcoólicas. Quanto ao tipo de bebida, 96,4\% dos participantes referiram consumir cerveja; $22,5 \%$, bebidas destiladas; $9,7 \%$, vinho.

Em relação ao padrão de uso de álcool, enquanto $31,9 \%$ dos participantes declararam-se abstêmios nos trinta dias prévios à entrevista, 
Distribuição das características sociodemográficas e ocupacionais de motoristas de caminhão abordados em rodovias do Estado de São Paulo, Brasil (N = 535), junho de 2012 a 25 de setembro de 2013.

\begin{tabular}{|c|c|c|c|c|}
\hline \multirow[t]{2}{*}{ Variáveis } & \multirow[t]{2}{*}{$\mathbf{n}$} & \multicolumn{2}{|c|}{ Total } & \multirow[t]{2}{*}{ Média ( \pm desvio padrão) } \\
\hline & & $\%$ & IC95\% & \\
\hline Idade (anos) & & & & $37,8( \pm 7,78)$ \\
\hline$\leq 36$ & 272 & 50,9 & & \\
\hline$>36$ & 262 & 49,1 & & \\
\hline Escolaridade (anos) & & & & $8,6( \pm 2,28)$ \\
\hline$\leq 9$ & 261 & 48,8 & & \\
\hline$>9$ & 274 & 51,1 & & \\
\hline \multicolumn{5}{|l|}{ Estado civil } \\
\hline Solteiro & 101 & 19,2 & $15,9-22,9$ & \\
\hline Casado/Amasiado & 392 & 74,7 & $70,7-78,3$ & \\
\hline Separado/Divorciado & 32 & 6,1 & $4,2-8,5$ & \\
\hline Anos de profissão & & & & $12,5( \pm 8,1)$ \\
\hline$\leq 10$ & 217 & 40,6 & & \\
\hline$>10$ & 318 & 59,4 & & \\
\hline \multicolumn{5}{|l|}{ Tipo de serviço } \\
\hline Contratado & 326 & 60,9 & $56,6-65,1$ & \\
\hline Autônomo & 209 & 39,1 & $34,9-43,3$ & \\
\hline \multicolumn{5}{|l|}{ Turno de trabalho } \\
\hline Diurno & 100 & 18,7 & $15,5-22,2$ & \\
\hline Diurno e noturno & 322 & 60,2 & $55,9-64,3$ & \\
\hline Noturno & 67 & 12,5 & $9,7-15,6$ & \\
\hline Outro & 46 & 8,6 & $6,3-11,2$ & \\
\hline Jornada diária (horas) & & & & $12,1( \pm 3,88)$ \\
\hline$\leq 12$ & 267 & 67,4 & & \\
\hline$>12$ & 126 & 32,1 & & \\
\hline Trajetória percorrida (km) & & & & $1.127,3( \pm 1.020,84)$ \\
\hline$\leq 750$ & 197 & 50,1 & & \\
\hline$>750$ & 196 & 49,9 & & \\
\hline Total & 535 & 100,0 & & \\
\hline
\end{tabular}

$34,6 \%$ (dos que haviam usado alguma quantidade de álcool nesse período) enquadraramse no padrão de uso pesado; $17,9 \%$, no padrão moderado; $15,6 \%$, no padrão leve de uso. Já $26 \%$ daqueles que beberam no último mês relataram ter ingerido álcool no padrão BPE, isto é, consumiram cinco ou mais doses alcoólicas em um período de duas horas e pelo menos uma vez no período citado. Soma-se a esse quadro o fato de que, conforme os critérios do ASSIST-WHO, identificou-se que $9,2 \%$ dos participantes não abstêmios estavam sob o risco, ao menos moderado, de desenvolver dependência de álcool (Tabela 2).

\section{Uso múltiplo de álcool e outras drogas}

O usuário de múltiplas drogas referiu-se ao uso de álcool associado a pelo menos uma substância psicoativa, tendo sido subdividido em uso múltiplo de drogas do tipo concorrente (CPU) ou simultâneo (SPU). Nesse sentido, 94,1\% dos participantes que já haviam experimentado álcool (uso na vida) relataram ter experimentado, também, alguma outra substância psicoativa (CPU), incluindo-se a cafeína dos energéticos nessa relação. O uso múltiplo de drogas do tipo CPU foi relatado, ainda, por $75,2 \%$ dos participantes que beberam nos 12 meses prévios à entrevista $\mathrm{e}$ por $53,6 \%$ daqueles que beberam no último mês. Dentre os que relataram ter feito CPU na vida, 
Tabela 2

Distribuição das variáveis sobre o consumo de álcool entre motoristas de caminhão abordados em rodovias do Estado de São Paulo, Brasil ( $\mathrm{N}=535)$, junho de 2012 a setembro de 2013.

\begin{tabular}{|c|c|c|c|c|}
\hline \multirow[t]{2}{*}{ Variáveis } & \multirow[t]{2}{*}{$\mathrm{n}$} & \multicolumn{2}{|c|}{ Total } & \multirow[t]{2}{*}{ Média ( \pm desvio padrão) } \\
\hline & & $\%$ & IC95\% & \\
\hline \multicolumn{5}{|l|}{ Uso na vida } \\
\hline Sim & 512 & 95,7 & $93,6-97,2$ & \\
\hline \multicolumn{5}{|l|}{ Uso no ano } \\
\hline Sim & 415 & 77,6 & $73,8-81,0$ & \\
\hline \multicolumn{5}{|l|}{ Uso no mês } \\
\hline Sim & 360 & 67,3 & $63,1-71,3$ & \\
\hline \multicolumn{5}{|l|}{ Frequência de uso no mês (vez/semana) } \\
\hline Menos de 1 & 137 & 38,7 & $33,6-44,0$ & \\
\hline Pelo menos 1 & 217 & 61,3 & $56,0-66,4$ & \\
\hline Número de doses por ocasião & & & & $6,4( \pm 6,43)$ \\
\hline$\leq 5$ & 212 & 59,0 & $53,7-64,2$ & \\
\hline$>5$ & 147 & 41,0 & $35,8-46,2$ & \\
\hline Número máximo de doses por ocasião & & & & $8,2( \pm 9,23)$ \\
\hline$\leq 5$ & 180 & 50,1 & $44,8-55,4$ & \\
\hline$>5$ & 179 & 49,9 & $44,6-55,2$ & \\
\hline \multicolumn{5}{|l|}{ Binge drinking } \\
\hline Sim & 93 & 26,0 & $21,5-30,8$ & \\
\hline \multicolumn{5}{|l|}{ Frequência de binge drinking } \\
\hline Mensalmente & 54 & 58,1 & $47,4-68,2$ & \\
\hline Semanalmente & 39 & 41,9 & $31,7-52,6$ & \\
\hline \multicolumn{5}{|l|}{ Padrão do uso de álcool (no mês) } \\
\hline Abstêmios & 166 & 31,9 & $27,9-36,1$ & \\
\hline Uso leve & 81 & 15,6 & $12,6-19,0$ & \\
\hline Uso moderado & 93 & 17,9 & $14,7-21,5$ & \\
\hline Uso pesado & 180 & 34,6 & $30,5-38,9$ & \\
\hline ASSIST-WHO (uso de risco) & & & & $4,1( \pm 3,89)$ \\
\hline Sem risco & 327 & 90,8 & $87,4-93,6$ & \\
\hline Com risco & 33 & 9,2 & $6,4-12,6$ & \\
\hline \multicolumn{5}{|l|}{ Bebe cerveja } \\
\hline Sim & 347 & 96,4 & $93,9-98,1$ & \\
\hline \multicolumn{5}{|l|}{ Bebe vinho } \\
\hline Sim & 35 & 9,7 & $6,9-13,3$ & \\
\hline \multicolumn{5}{|l|}{ Bebe destilado } \\
\hline Sim & 81 & 22,5 & $18,3-27,2$ & \\
\hline Total & 535 & 100,0 & & \\
\hline
\end{tabular}

ASSIST-WHO: Teste de Triagem do Envolvimento com Álcool, Cigarro e Outras Substâncias.

$54,6 \%$ já haviam feito uso múltiplo de drogas do tipo SPU, assim como $66 \%$ dos que fizeram CPU no ano e $76,2 \%$ dos que fizeram CPU nos trinta dias prévios à entrevista. Portanto, observou-se que quanto mais recente o engajamento em uso múltiplo de drogas do tipo CPU, maior seria a probabilidade de ter feito esse uso na mesma ocasião de consumo (SPU). Os energéticos, anfe- taminas e cigarro despontaram como as substâncias psicoativas mais frequentemente usadas entre pessoas que relataram beber (CPU), enquanto cigarro, cocaína e energéticos foram as substâncias mais comumente usadas simultaneamente a álcool (SPU) (Tabela 3). 
Distribuição das variáveis sobre o uso múltiplo de álcool e outras drogas, dos tipos concorrente (CPU) e simultâneo (SPU), para as medidas de uso na vida, no ano e no mês, entre motoristas de caminhão abordados em rodovias do Estado de São Paulo, Brasil ( $N=535)$, junho de 2012 a setembro de 2013

\begin{tabular}{|c|c|c|c|c|c|c|}
\hline \multirow[t]{2}{*}{ CPU } & \multicolumn{2}{|c|}{ Vida } & \multicolumn{2}{|c|}{ Ano } & \multicolumn{2}{|c|}{ Mês } \\
\hline & $\mathrm{n}$ & $\%$ & $\mathbf{n}$ & $\%$ & $\mathbf{n}$ & $\%$ \\
\hline Pelo menos uma substância & 482 & 94,1 & 312 & 75,2 & 193 & 53,6 \\
\hline Bebidas energéticas & 433 & 84,6 & 253 & 61,0 & 122 & 33,9 \\
\hline Anfetaminas & 301 & 58,8 & 126 & 30,4 & 55 & 15,3 \\
\hline Tabaco & 237 & 46,3 & 100 & 24,1 & 80 & 22,2 \\
\hline Maconha & 122 & 23,8 & 11 & 2,7 & 5 & 1,4 \\
\hline Cocaína & 97 & 19,0 & 34 & 8,2 & 8 & 2,3 \\
\hline Crack & 10 & 2,0 & - & - & - & - \\
\hline Metanfetamina & 6 & 1,2 & - & - & - & - \\
\hline \multirow[t]{2}{*}{ SPU } & \multicolumn{2}{|c|}{ Vida } & \multicolumn{2}{|c|}{ Ano } & \multicolumn{2}{|c|}{ Mês } \\
\hline & n & $\%$ & $\mathbf{n}$ & $\%$ & $\mathbf{n}$ & $\%$ \\
\hline Pelo menos uma substância & 263 & 54,6 & 206 & 66,0 & 147 & 76,2 \\
\hline Bebidas energéticas & 148 & 34,2 & 79 & 31,2 & 36 & 29,5 \\
\hline Anfetaminas & 33 & 11,0 & 8 & 6,3 & 1 & 1,8 \\
\hline Tabaco & 143 & 60,3 & 79 & 79,0 & 60 & 75,0 \\
\hline Maconha & 35 & 28,7 & 4 & 36,4 & 1 & 20,0 \\
\hline Cocaína & 41 & 42,3 & 17 & 50,0 & 3 & 37,5 \\
\hline Crack & 2 & 20,0 & - & - & - & - \\
\hline Metanfetamina & - & - & - & - & - & - \\
\hline
\end{tabular}

Padrão de uso de álcool entre participantes que relataram usar apenas álcool e aqueles que fizeram uso múltiplo de drogas

Com base nesses resultados, foi importante identificar se o padrão de uso de álcool seria influenciado pelo uso múltiplo de drogas, de tal forma que, para isso, os participantes foram agrupados em: (a) usuários apenas de álcool (álcool), (b) uso múltiplo de drogas do tipo CPU e (c) uso múltiplo de drogas do tipo SPU. Dessa forma, identificou-se diferença do número de doses alcoólicas consumidas por ocasião, nos últimos trinta dias, entre os grupos ( $\mathrm{p}<0,01)$, assim como uma diferença quanto ao número máximo de doses alcoólicas consumidas por ocasião $(\mathrm{p}<$ 0,001 ) e padrão do uso de álcool nos últimos trinta dias $(\mathrm{p}<0,001)$. Uma tendência de diferença entre os grupos foi observada quando avaliado o risco de desenvolvimento de dependência de álcool, de acordo com a pontuação do ASSISTWHO. Posteriormente, os grupos (álcool; CPU; SPU) foram comparados, dois a dois, para que diferenças intergrupos, quanto às variáveis con- sideradas, fossem identificadas. Nesse caso, não foi identificada diferença entre os participantes que relataram consumir apenas álcool e aqueles que houvessem feito uso múltiplo de drogas do tipo CPU para nenhuma das variáveis. Em contrapartida, os usuários que consumiram apenas álcool relataram usar menos doses alcoólicas por ocasião ( $\mathrm{p}<0,001)$, menor número máximo de doses alcoólicas por ocasião $(p<, 001)$, menor pontuação no ASSIST-WHO ( $p<0,001)$ e, ainda, apresentaram padrão de uso de álcool menos pesado $(\mathrm{p}<0,001)$ em relação ao uso múltiplo de drogas do tipo SPU. Igualmente, os usuários do tipo CPU relataram consumir menos doses alcoólicas por ocasião ( $\mathrm{p}=0,05)$, menor número máximo de doses alcoólicas por ocasião $(\mathrm{p}<$ $0,01)$ e padrão menos pesado de uso $(\mathrm{p}<0,01)$, do que usuários do tipo uso múltiplo de drogas do tipo SPU. Finalmente, não houve diferença entre os grupos CPU e SPU quanto à pontuação no ASSIST-WHO ( $\mathrm{p}=0,1895$ ) (Tabelas 4 e 5). 
Tabela 4

Padrão de uso de álcool entre usuários exclusivos de álcool (álcool) e aqueles que associam o uso de álcool a pelo menos uma outra droga, do tipo concorrente (CPU) ou simultâneo (SPU), entre motoristas de caminhão que relataram usar álcool nos trinta dias prévios à entrevista, abordados em rodovias do Estado de São Paulo, Brasil ( $n=359)$, junho de 2012 a setembro de 2013.

\begin{tabular}{|c|c|c|c|c|}
\hline \multirow[t]{2}{*}{ Variáveis } & \multirow{2}{*}{$\begin{array}{c}\text { Álcool } \\
\text { Média ( } \pm \text { desvio padrão) }\end{array}$} & \multirow{2}{*}{$\begin{array}{c}\text { CPU } \\
\text { Média ( } \pm \text { desvio padrão) }\end{array}$} & \multicolumn{2}{|c|}{ SPU } \\
\hline & & & Média ( \pm desvio padrão) & Valor de $\mathrm{p}$ \\
\hline Número de doses por ocasião & $4,9 \pm 4,07$ & $6,5 \pm 8,53$ & $7,6 \pm 7,50$ & $<0,01^{*}$ \\
\hline Número máximo doses por ocasião & $6,1 \pm 5,77$ & $7,0 \pm 8,41$ & $10,5 \pm 11,78$ & $<0,001^{\star}$ \\
\hline \multirow[t]{2}{*}{ ASSIST-WHO } & $4,9 \pm 3,35$ & $5,3 \pm 4,02$ & $5,9 \pm 4,23$ & 0,057 \\
\hline & $\%$ & $\%$ & $\%$ & Valor de $\mathrm{p}$ \\
\hline \multicolumn{5}{|l|}{ Binge drinking } \\
\hline $\operatorname{Sim}$ & 21,8 & 19,6 & 28,8 & 0,297 \\
\hline \multicolumn{5}{|l|}{ Padrão de uso de álcool (no mês) } \\
\hline Uso leve & 29,4 & 39,1 & 14,7 & $<0,001^{\star}$ \\
\hline Uso moderado & 29,4 & 15,2 & 25,9 & \\
\hline Uso pesado & 41,3 & 45,6 & 59,5 & \\
\hline Total (n) & 166 & 46 & 146 & \\
\hline
\end{tabular}

ASSIST-WHO: Teste de Triagem do Envolvimento com Álcool, Cigarro e Outras Substâncias.

* $\mathrm{p}<0,05$.

Tabela 5

Valores de p para a comparação entre os entrevistados que relataram consumir exclusivamente álcool nos últimos trinta dias ou fizeram uso múltiplo de drogas do tipo concorrente (CPU) ou simultâneo (SPU), quanto ao número de doses alcoólicas, número máximo de doses alcoólicas, Teste de Triagem do Envolvimento com Álcool, Cigarro e Outras Substâncias (ASSISTWHO) e padrão de uso de álcool, em rodovias do Estado de São Paulo, Brasil ( $=$ 359), junho de 2012 a setembro de 2013.

\begin{tabular}{lcccc}
\hline & Doses & Máximo de doses & ASSIST-WHO & Padrão \\
\hline Álcool vs. CPU & 0,836 & 0,824 & 0,682 & 0,159 \\
Álcool vs. SPU & $<0,001$ & $<0,001$ & $<0,001$ & $<0,001$ \\
CPU vs. SPU & 0,050 & $<0,01$ & 0,190 & $<0,01$ \\
\hline
\end{tabular}

\section{Discussão}

Os motoristas abordados eram, em sua maioria, jovens, de baixa escolaridade, que viviam em situação marital. Em termos ocupacionais, os participantes tinham larga experiência como motoristas profissionais, com excessiva carga diária de trabalho, percorrendo longas distâncias. Esses dados são consistentes com os de estudos prévios, particularmente no que diz respeito a idade 23,24,25, estado civil 23,25, anos de experiência 26,27 , carga diária de trabalho $26,28,29,30$ e turno de trabalho 26 .
A prevalência de uso experimental de álcool foi de $95,7 \%$, de $77,6 \%$ para o uso no ano e $67,3 \%$ no mês. Especificamente em relação ao uso experimental de álcool, no presente trabalho a prevalência identificada entre os motoristas de caminhão foi superior à previamente relatada para a população geral brasileira $(74,6 \%) 31$, sendo superior também à prevalência detectada entre homens brasileiros de 35 anos ou mais $(86,1 \%)^{31}$, faixa etária condizente com a idade desta amostra.

Ainda, de acordo com outro levantamento estatístico, $65 \%$ dos homens brasileiros de 18 anos ou mais relataram ter usado álcool 
nos 12 meses prévios à entrevista 5, assim como $67 \%$ de uma amostra de homens residentes do Estado de São Paulo 18, sugerindo que a prevalência de uso de álcool no ano entre os motoristas de caminhão abordados $(77,6 \%)$ seja superior à identificada em uma amostra da população nacional e em outra específica do Estado de São Paulo. O mesmo resultado foi observado para a medida de uso de álcool no mês, ou seja, enquanto $61,3 \%$ dos motoristas de caminhão desta pesquisa relataram beber com frequência semanal no mínimo, nos últimos trinta dias, 39\% dos homens brasileiros relataram fazê-lo 5. Em relação a amostras de motoristas de outros estudos, $71,1 \%$ dos motoristas profissionais e privados do país, abordados em um levantamento estatístico nacional 32 , relataram ter usado álcool no ano, próximo à prevalência aqui identificada de 77,6\%.

Em particular sobre o padrão de uso de álcool, identificou-se que $34,6 \%$ dos participantes fizeram uso pesado da substância, prevalência superior aos 21,5\% entre os homens, de 35 a 49 anos, residentes do Estado de São Paulo 19. A diferença torna-se ainda mais notável ao se considerar que aquele estudo (GENACIS) 19 usou, como medida de uso de álcool, os últimos 12 meses prévios à entrevista, enquanto na presente pesquisa foi utilizada a medida de uso no último mês.

Ainda, identificou-se que $26 \%$ dos participantes relataram ter bebido no padrão BPE no mês que antecedeu a entrevista, o que corrobora os dados de Pechansky et al. 32 , que apontaram que $25,5 \%$ dos motoristas abordados por um levantamento estatístico nacional relataram uso no padrão BPE pelo menos mensalmente. Diante do exposto, é importante observar que o uso em BPE pelos participantes desta pesquisa pode ter sido superior ao previamente identificado para a população geral brasileira, uma vez que $40 \%$ dos brasileiros com mais de 18 anos se enquadram no padrão BPE no ano 5, assim como 15,4\% dos homens do Estado de São Paulo 33.

A média da pontuação do ASSIST-WHO entre os participantes foi de $4,1( \pm 3,89)$, dentre os quais $9,2 \%$ estariam sob o risco ao menos moderado de desenvolverem dependência de álcool. Esses critérios são indicativos de uso nocivo de álcool, ou seja, prejudicial a algum domínio da vida do usuário 34. Nesse sentido, quando comparados aos $5 \%$ dos brasileiros que fazem uso nocivo de álcool 5 , observa-se que os participantes da presente pesquisa têm maior prevalência de risco, se comparados com a população geral brasileira, para desenvolver dependência de álcool.

Sobre o uso múltiplo de drogas, 53,6\% dos participantes relataram ter consumido álcool e pelo menos outra substância psicoativa (CPU) no mês prévio à entrevista, dos quais $76,2 \%$ o fizeram na forma de SPU. Não há estudos que tenham abordado esse tema na população geral brasileira, a não ser um levantamento estatístico realizado com estudantes universitários, o qual identificou que $17 \%$ de uma amostra nacional de estudantes relatou fazer uso múltiplo de drogas, do tipo CPU, no mês prévio à entrevista; destes, 35\% usaram mais de duas substâncias juntas ou em proximidade temporal (SPU) 10 .

Em face desse cenário, preocupa observar que o padrão do uso de álcool entre os motoristas de caminhão investigados nesta pesquisa seja superior ao já identificado para a população geral brasileira e também do Estado de São Paulo. Preocupa ainda mais constatar a existência do uso múltiplo de drogas entre eles.

É possível que tal padrão de uso de álcool e de outras drogas entre esses motoristas possa ser uma estratégia para suportar as condições insalubres de sua profissão 17,35. Ademais, especula-se que esse uso possa ser uma medida alternativa após o controle exercido sobre a produção, prescrição e comercialização das anfetaminas, de acordo com o estipulado pela Resolução $R D C$ no 52/2011 da Agência Nacional de Vigilância Sanitária 36 .

Sobretudo, é preocupante notar que o uso de álcool afeta a direção mesmo após poucas doses 37 e que afeta também condutores sóbrios que tenham o costume de beber excessivamente 38 , indicando a inexistência de um nível seguro de uso de álcool quando se pensa em associá-lo à direção. No cenário descrito anteriormente, causa preocupação, ainda, observar que a problemática do beber e dirigir pode piorar sob a condição de uso múltiplo de drogas, uma vez que se identificou que motoristas que relataram seguir este padrão bebiam mais e de forma mais pesada que aqueles que não se enquadravam nesse tipo de uso.

O uso múltiplo de drogas, por si só, já tem sido identificado como agravante para o acontecimento de comportamentos de risco ou acidentes no contexto do trânsito, inclusive com o acometimento de vítimas de morte 38,39,40. Estudo retrospectivo conduzido na Suécia 40 avaliou as concentrações de álcool e de outras drogas em 895 amostras de sangue de motoristas que morreram em acidentes de trânsito, tendo sido identificado que 1,8\% deles haviam consumido álcool e alguma droga ilícita; 3,5\%, álcool e algum medicamento prescrito; e, finalmente, $0,9 \%$ havia consumido álcool + droga ilícita + medicamento. Em adição, tem sido apontado que o uso múltiplo de drogas é indicativo de maiores chances de desenvolvimento de dependência 11 . 
Quanto ao tipo de uso múltiplo de drogas, a combinação entre bebidas alcoólicas e energéticas foi a mais prevalente entre os participantes da presente pesquisa. Como essa mistura atenua a percepção dos efeitos sedativos e dos prejuízos psicomotores causados pelo álcool 41, aumenta a chance de os condutores envolverem-se em comportamentos de risco no trânsito, como dirigir em velocidades maiores que as permitidas nas vias públicas, o que os torna vulneráveis ao acontecimento de acidentes de trânsito e outros desfechos negativos 12 .

Portanto, destacamos a necessidade de mais dados empíricos para a compreensão da relação entre o uso de drogas no padrão uso múltiplo e a ocorrência de acidentes de trânsito, assim como encorajamos a fiscalização da obediência às leis que regulamentam e coíbem o uso de álcool e outras drogas no trânsito. Também acreditamos ser necessário estabelecer medidas que identifiquem, com fins de prevenção, motoristas que estejam abusando ou já estejam dependentes de álcool ou outras drogas, pois está claro na lite- ratura que motoristas em uso pesado de álcool são reincidentes quanto ao comportamento de beber e dirigir 7, causando algum tipo de ônus a si mesmos ou à sociedade como um todo.

Embora o presente estudo seja inédito, visto que identificou o padrão de uso de álcool e de uso múltiplo de álcool e outras substâncias entre motoristas de caminhão, possibilitando melhor compreensão sobre o tema, é importante comentar algumas das suas limitações: (a) dada a característica de estudo transversal, esta investigação é apenas um recorte da situação; (b) o uso de amostra não probabilística impede que os dados sejam generalizados para toda a população brasileira de motoristas de caminhão; (c) não se pode afirmar que os participantes que relataram o uso de álcool e outras drogas tenham consumido tais substâncias enquanto dirigiam; (d) finalmente, a comparação da prevalência de álcool e outras drogas com a encontrada em outros estudos é limitada, já que as referidas pesquisas utilizaram metodologias específicas. 


\section{Resumen}

Los accidentes de tráfico han causado más de un millón de muertes en todo mundo. Brasil tiene una de las tasas más altas de ese indicador. En este trabajo se intentó estimar cómo el consumo de alcohol es un factor etiológico para los accidentes de tráfico. Se analizó el patrón de consumo de alcohol y su asociación con otras drogas en una muestra de conveniencia de 684 conductores de camiones en el Estado de São Paulo, Brasil. Treinta días previos a la entrevista, un 67,3\% de los participantes consumieron alcohol, un 34,6\% de forma abusiva, un $26 \%$ como binge drinking y un 9,3\% estaban bajo riesgo de generar una adicción. De ellos, un 54,6\% presentaron policonsumo de drogas, frecuentemente alcohol y tabaco o bebidas energéticas. Los participantes que presentaron un policonsumo de alcohol y otras drogas mostraron un consumo de alcohol más abusivo que los que informaron sobre un consumo exclusivo de alcohol. En general, el consumo de alcohol es un problema, teniendo en cuenta el contexto del tráfico y puede empeorar aún más con el policonsumo. Por lo tanto, los autores sugieren el control de esta cuestión, ya que tal consumo genera problemas potencialmente graves para la salud del consumidor, así como de la sociedad que le circunda.

Accidentes de Tránsito; Alcoholismo; Bebidas Alcohólicas; Estudos Transversales

\section{Colaboradores}

K. Leopoldo e L. G. Oliveira participaram da concepção do trabalho, da aquisição e da análise dos dados, assim como do esboço, da elaboração e da aprovação final do artigo. V. Leyton colaborou na concepção do trabalho e na aquisição dos dados, assim como da aprovação final do artigo. Todos os autores são responsáveis por todos os aspectos do trabalho nos quesitos de acurácia e integridade.

\section{Agradecimentos}

Os autores agradecem o apoio financeiro da FAPESP (processos no 2011/11682-0; 2012/00973-6; 2012/22569-2); às entidades civis Serviço Social do Transporte (SEST) e Serviço Nacional de Aprendizagem do Transporte (SENAT), pela autorização de uso de três de seus postos de atendimento para o acesso à população-alvo; e, finalmente, ao LIM-40 da Faculdade de Medicina, Universidade de São Paulo.

\section{Referências}

1. World Health Organization. Global status report on road safety 2013: supporting a decade of action. Geneva: World Health Organization; 2013.

2. Departamento de Estradas de Rodagem do Paraná. Década de ação para a segurança no trânsito 2011-2020. http://www.der.pr.gov.br/modules/ conteudo/conteudo.php?conteudo=220 (acessado em 17/Nov/2014)

3. Instituto de Pesquisa Econômica Aplicada; Departamento Nacional de Trânsito. Impactos sociais e econômicos dos acidentes de trânsito nas rodovias brasileiras. Brasília: Instituto de Pesquisa Econômica Aplicada/Departamento Nacional de Trânsito; 2006.
4. World Health Organization. Global status report on alcohol and health. Geneva: World Health Organization; 2014.

5. Laranjeira R, Pinsky I, Zaleski M, Caetano R. I levantamento nacional sobre os padrões de consumo de álcool na população brasileira. Brasília: Secretaria Nacional de Políticas sobre Drogas, Ministério da Justiça; 2007.

6. Girotto E, Mesas AE, Andrade SM, Birolim MM. Psychoactive substance use by truck drivers: a ystematic review. Occup Environ Med 2014; 71:71-6.

7. Holmgren A, Holmgren P, Kugelberg FC, Jones AW, Ahlner J. High re-arrest rates among drug-impaired drivers despite zero-tolerance legislation. Accid Anal Prev 2008; 40:534-40. 
8. Duarte PCAV, Stempliuk V, Barroso LP. Relatório brasileiro sobre drogas. Brasília: Secretaria Nacional de Políticas sobre Drogas, Ministério da Justiça; 2009.

9. Martin CS. Timing of alcohol and other drug use. Alcohol Health Res World 2008; 31:96.

10. Oliveira LG, Alberghini DG, Santos BD, Andrade AG. Polydrug use among college students in Brazil: a nationwide survey. Rev Bras Psiquiatr 2013; 35:221-30.

11. Associação Brasileira de Psiquiatria. Abuso e dependência de múltiplas drogas, 2012. http://www. projetodiretrizes.org.br/projeto_diretrizes2013/ drogas.pdf (acessado em 17/Nov/2014).

12. Eckschmidt F, Andrade AG, Santos B, Oliveira LG. The effects of alcohol mixed with energy drinks (AmED) on traffic behaviors among Brazilian college students: a national survey. Traffic Inj Prev 2013; 14:671-9.

13. Oliveira LG, Endo LG, Sinagawa DM, Yonamine M, Munoz DR, Leyton V. A continuidade do uso de anfetaminas por motoristas de caminhão no Estado de São Paulo, Brasil, a despeito da proibição de sua produção, prescrição e uso. Cad Saúde Pública 2013; 29:1903-9.

14. United Nations. 74th plenary meeting. New York: United Nations; 2010.

15. Presidência da República. Lei no 11.705, de 19 de junho de 2008. Altera a Lei no 9.503, de 23 de setembro de 1997, que "institui o Código de Trânsito Brasileiro", e a Lei no 9.294, de 15 de julho de 1996, que dispõe sobre as restrições ao uso e à propaganda de produtos fumígeros, bebidas alcoólicas, medicamentos, terapias e defensivos agrícolas, nos termos do $\$ 4$ o do art. 220 da Constituição Federal, para inibir o consumo de bebida alcoólica por condutor de veículo automotor, e dá outras providências. Diário Oficial da União 2008; 20 jun.

16. Presidência da República. Lei no 12.760 , de 20 de dezembro de 2012. Altera a Lei no 9.503, de 23 de setembro de 1997, que institui o Código de Trânsito Brasileiro. Diário Oficial da União 2012; 21 dez.

17. Leyton V, Sinagawa DM, Oliveira K, Schmitz W, Andreuccetti G, De Martinis BS, et al. Amphetamine, cocaine and cannabinoids use among truck drivers on the roads in the State of São Paulo, Brazil. Forensic Sci Int 2012; 215:25-7.

18. United Nations on Drugs and Crime. World drug report 2014. New York: United Nations; 2014.

19. Kerr-Corrêa F, Hegedus AM, Trinca LA, Tucci AM, Kerr-Pontes LR, Sanches AF, et al. Differences in drinking patterns between men and women in Brazil. In: Obot IS, Room R, editors. Alcohol, gender and drinking problems: perspectives from low and middle income countries. Geneva: World Health Organization, 2005. p. 49-68.

20. National Institute on Alcohol and Alcoholism. Moderate \& binge drinking. http://www.niaaa.nih. gov/alcohol-health/overview-alcohol-consump tion/moderate-binge-drinking (acessado em 17/ Nov/2014).

21. World Health Organization. The Alcohol, Smoking and Substance Involvement Screening Test (ASSIST): manual for use in primary care. Geneva: World Health Organization; 2010.
22. European Monitoring Centre for Drugs and Drug Addiction. Polydrug use: patterns and responses. Luxembourg: Office for Official Publications of the European Communities; 2009.

23. Yonamine M, Sanches LR, Paranhos BA, Almeida RM, Andreuccetti G, Leyton V. Detecting alcohol and illicit drugs in oral fluid samples collected from truck drivers in the state of São Paulo, Brazil. Traffic Inj Prev 2013; 14:127-31.

24. Lemos LC, Marqueze EC, Sachi F, Lorenzi-Filho G, Moreno CRC. Síndrome da apnéia obstrutiva do sono em motoristas de caminhão. J Bras Pneumol 2009; 35:500-6.

25. Silva-Junior FP, Pinho RSN, Mello MT, Bruin VMS, Bruin PFC. Risk factors for depression in truck drivers. Soc Psychiatry Psychiatr Epidemiol 2009; 44:125-9.

26. Ulhoa MA, Marqueze EC, Lemos LC, Silva LG, Silva AA, Nehme P, et al. Minor psychiatric disorders and working conditions in truck drivers. Rev Saúde Pública 2010; 44:1130-6.

27. Pinho RS, Silva-Júnior FP, Bastos JP, Maia WS, Mello MT, Bruin VM, et al. Hypersomnolence and accidents in truck drivers: a cross-sectional study. Chronobiol Int 2006; 23:963-71.

28. Oliveira LG, Santos B, Goncalves PD, Carvalho HB, Massad E, Leyton V. Attention performance among Brazilian truck drivers and its association with amphetamine use: pilot study. Rev Saúde Pública 2013; 47:1001-5.

29. Oliveira LG, Yonamine M, Andreucceti G, Ponce JC, Leyton V. Alcohol and other drug use by Brazilian truck drivers: a cause for concern? Rev Bras Psiquiatr 2012; 34:116-7.

30. Penteado RZ, Gonçalves CGO, Costa DD, Marques JM. Trabalho e saúde em motoristas de caminhão no interior de São Paulo. Saúde Soc 2008; 17:35-45.

31. Carlini EA, Galduróz JCF, Noto AR, Fonseca AM, Carlini CM, Oliveira LG, et al. II levantamento domiciliar sobre o uso de drogas psicotrópicas no Brasil: estudo envolvendo as 108 maiores cidades do País. Brasília: Secretaria Nacional de Políticas sobre Drogas, Ministério da Justiça; 2005.

32. Pechansky F, Duarte P, Boni R. Uso de bebidas alcoólicas e outras drogas nas rodovias brasileiras e outros estudos. Brasília: Secretaria Nacional de Políticas sobre Drogas, Ministério da Justiça; 2010.

33. Silveira CM, Wang YP, Andrade AG, Andrade LH. Heavy episodic drinking in the São Paulo epidemiologic catchment area study in Brazil: gender and sociodemographic correlates. J Stud Alcohol Drugs 2007; 68:18-27.

34. Observatório Brasileiro de Informações sobre Drogas. Informações sobre drogas: padrões de uso. http://www.obid.senad.gov.br/portais/OBID/ conteudo/index.php?id_conteudo $=11251 \&$ rastr o=INFORMA\%C3\%87\%C3\%95ES+SOBRE+DRO GAS/Padr\%C3\%B5es+de+uso (acessado em 17/ Nov/2014).

35. Takitane J, Oliveira LG, Endo LG, Oliveira KCBG, Muñoz DR, Yonamine M, et al. Uso de anfetaminas por motoristas de caminhão em rodovias do Estado de São Paulo: um risco à ocorrência de acidentes de trânsito? Ciênc Saúde Coletiva 2013; 18:1247-54 
36. Agência Nacional de Vigilância Sanitária. Resolução RDC no 52, de 6 de outubro de 2011. Dispõe sobre a proibição do uso das substâncias anfepramona, femproporex e mazindol, seus sais e isômeros, bem como intermediários e medidas de controle da prescrição e dispensação de medicamentos que contenham a substância sibutramina, seus sais e isômeros, bem como intermediários e dá outras providências. Diário Oficial da União 2011; 7 out.

37. Gonçalves PD, Cunha PJ, Malbergier A, Amaral RA, Oliveira LG, Yang JJ, et al. The association between low alcohol use and traffic risk behaviors among Brazilian college students. Alcohol 2012; 46:673-9.

38. Karjalainen K, Lintonen T, Impinen A, Lillsunde $\mathrm{P}$, Ostamo A. Poly-drug findings in drugged driving cases during 1977-2007. J Subst Use 2010; 15:143-56.
39. Legrand SA, Silverans P, Paepe P, Buylaert W, Verstraete AG. Presence of psychoactive substances in injured Belgian drivers. Traffic Inj Prev 2013; 14:461-8.

40. Ahlner J, Holmgren A, Jones AW. Prevalence of alcohol and other drugs and the concentrations in blood of drivers killed in road traffic crashes in Sweden. Scand J Public Health 2014; 42:177-83.

41. Ferreira SE, Mello MT, Pompéia S, de Souza-Formigoni ML. Effects of energy drink ingestion on alcohol intoxication. Alcohol Clin Exp Res 2006; 30:598-605.

Recebido em 25/Mar/2014

Versão final reapresentada em 17/Nov/2014

Aprovado em 02/Mar/2015 